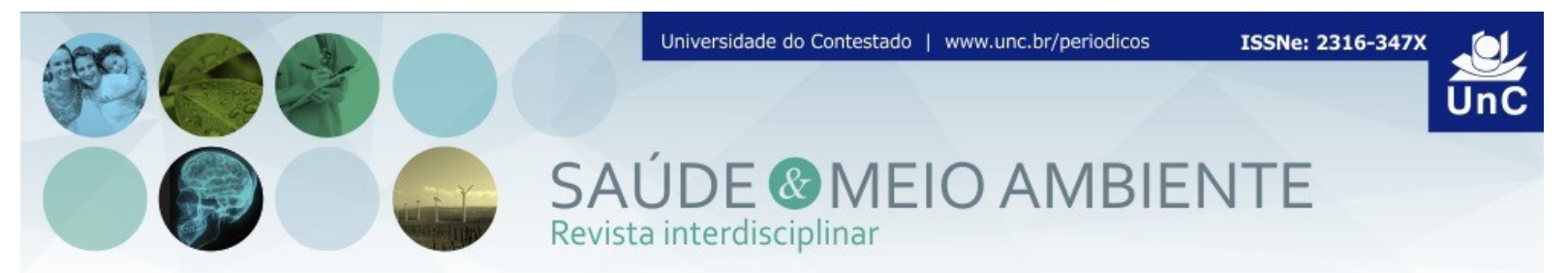

\title{
CONHECIMENTO POPULAR SOBRE PLANTAS MEDICINAIS UTILIZADAS NO MUNICÍPIO DE MAFRA, SC, BRASIL
}

\author{
Thais Humenhuk ${ }^{1}$ \\ Daniela Regina Buch Leite ${ }^{2}$ \\ Mário Fritsch ${ }^{3}$
}

\begin{abstract}
RESUMO
O conhecimento popular sobre plantas medicinais, apesar de ser bem disseminado no Brasil, está sendo perdido devido à globalização. Além disso, e apesar de apresentar vantagens, o uso de plantas de maneira errônea pode causar danos à saúde dos usuários. O presente estudo teve como objetivo apontar algumas plantas utilizadas no município e resgatar uma pequena parcela do conhecimento popular, comparando as informações do senso comum com o conhecimento científico sobre essas plantas. O levantamento etnobotânico ocorreu entre maio e junho de 2019 , através de entrevistas semiestruturadas com a população do município de Mafra (SC). Após a coleta dos dados, foram realizadas buscas na literatura sobre as espécies mencionadas pelos entrevistados. Dos 22 entrevistados, foi observado a prevalência de entrevistados do sexo feminino (86,36 \%). Além disso, a maior parte dos participantes possuem mais de 60 anos de idade $(36,36 \%)$ e ensino fundamental incompleto $(22,72 \%)$. A fonte principal do conhecimento popular sobre o uso das plantas foi transmitida pelos seus antepassados. Alguns informantes $(18,18 \%)$ relataram o aparecimento de efeitos adversos com o uso de algumas espécies. Foram mencionadas 87 plantas distribuídas em 49 famílias, sendo as famílias dominantes Asteraceae (11,5\%), Lamiaceae (10,3\%). As plantas mais citadas foram: babosa (Aloe sp.), camomila (Chamomilla sp.), capim-limão (Cymbopogon sp.), gervão (Stachytarpheta $s p$.$) , hortelã (Mentha s p$.) e penicilina (Alternanthera sp.). Com esses resultados, foi possível mapear o uso e levantar dados acerca de algumas plantas medicinais utilizadas pela população de Mafra e concluir que a maioria das espécies do uso popular apresentam a mesma indicação encontrada na literatura científica.
\end{abstract}

Palavras-Chave: Etnomedicina. Etnobotânica. Espécies terapêuticas. Medicina popular.

\footnotetext{
${ }^{1}$ Graduanda em Farmácia. Universidade do Contestado. Campus Mafra. Santa Catarina. Brasil. Email: thais.humenhuk@outlook.com

${ }^{2}$ Mestre em Biologia Celular e Molecular pela Universidade Federal do Paraná. Docente da Universidade do Contestado, Campus Mafra. Santa Catarina. Brasil. E-mail:

buchdaniela84@gmail.com

${ }^{3}$ Mestre em Botânica pela Universidade Federal do Paraná. Docente da Universidade do Contestado, Campus Mafra. Santa Catarina. Brasil. E-mail: mariof@unc.br
} 
POPULAR KNOWLEDGE ABOUT MEDICINAL PLANTS USED IN MAFRA, SC, BRAZIL

\begin{abstract}
Although popular knowledge about medicinal plants is widespread in Brazil, it is being lost due to globalization. In addition, despite having advantages, the erroneous use of plants can cause damage to the health of users. The present study aimed to point out some plants used in the municipality. It also aims to rescue a small portion of regional popular knowledge and compare common sense information with scientific knowledge about medicinal plants. The ethnobotanical survey took place between May and June 2019, through semi-structured interviews with the population of the municipality of Mafra (SC). After data collection, literature searches were performed on the species mentioned by the interviewees. Among the 22 respondents, was observed a predominance of women (86.36\%). In addition, most participants are over 60 years old $(36.36 \%)$ and have incomplete elementary school $(22.72 \%)$. The main source of popular knowledge about plant use was transmitted by ancestors. Some informants $(18.18 \%)$ reported the appearance of adverse effects with the use of some species. Eighty-six plants distributed in 49 families were mentioned, the dominant ones being Asteraceae (11.5\%) and Lamiaceae (10.3\%). The most cited plants were: babosa (Aloe sp.), camomila (Chamomilla sp.), capim-limão (Cymbopogon sp.), gervão (Stachytarpheta $s p$.$) , hortelã (Mentha sp.) e penicilina (Alternanthera s p$. .). With these results, it was possible to map the use and collect data about some medicinal plants used by the population of Mafra and conclude that most species of popular use have the same indication found in the scientific literature.
\end{abstract}

Keywords: Ethnomedicine. Ethnobotany. Therapeutic species. Popular medicine.

\title{
INTRODUÇÃO
}

No Brasil, a população possui uma longa tradição no uso de plantas medicinais para o tratamento de diversas patologias, fato que tem chamado a atenção de pesquisadores para o estudo mais detalhado sobre as espécies ${ }^{(1)}$. O conhecimento tradicional brasileiro sobre plantas tem influência de várias culturas distintas, como colonizadores europeus, grupos nativo-americanos e africanos, sendo esse conhecimento passado hereditariamente para várias gerações ${ }^{(2)}$. Apesar do conhecimento popular ter sido bem disseminado no país, alguns fatores podem comprometer a dispersão do conhecimento popular e a preservação das espécies de plantas. Exemplo desses fatores são a redução das áreas naturais e a desvalorização dos saberes tradicionais pelas novas gerações ${ }^{(3)}$.

Para a restauração desse conhecimento surgiu a etnobotânica, definida como o estudo das relações entre povos e plantas ${ }^{(4)}$ somando-se os fatores ambientais e culturais $^{(5)}$, que tem sido empregada como ponto de partida para projetos de desenvolvimento de medicamentos nas últimas décadas ${ }^{(6)}$. Um levantamento etnobotânico( $^{(7)}$ enfatizou a importância do estudo da região da Mata Atlântica, 
apresentando as espécies medicinais mais utilizadas por comunidades rurais próximas. Uma região, destacada por sua relevância ecológica da biodiversidade ${ }^{(8)}$ e que faz parte do bioma Mata Atlântica é o Planalto Norte Catarinense. Todavia, até o momento não existiam dados sobre as plantas empregadas na medicina popular nessa região, dados que poderiam auxiliar na restauração do conhecimento tradicional e das espécies mais utilizadas.

Por esses motivos, o estudo foi realizado no município de Mafra, localizado no Planalto Norte Catarinense, com o objetivo de apresentar as plantas mais utilizadas no município, visando resgatar uma pequena parcela do conhecimento popular regional e comparar as informações do senso comum como as do conhecimento científico sobre as plantas medicinais.

\section{MATERIAIS E MÉTODOS}

O estudo foi desenvolvido no município de Mafra, localizado no Planalto Norte do estado de Santa Catarina a $26^{\circ} 06^{\prime} 50^{\prime \prime}$ S e 49 48'38" O (Figura 1), que possui população estimada de 56.017 habitantes e área territorial de $1.404,084$ km$^{2(9)}$.

O levantamento de informações sobre o uso popular de plantas para uso medicinal e a coleta de amostras das espécies medicinais ocorreu entre maio e junho de 2019, por meio de uma pesquisa de campo qualitativa. Com o questionário semiestruturado para a coleta de dados, foram feitas entrevistas aplicadas em uma amostra de 22 pessoas selecionadas a partir do hábito de utilizar plantas na terapêutica. Os primeiros entrevistados convidados a participar da pesquisa foram familiares e vizinhos, os quais indicaram outras pessoas.

O questionário foi composto de perguntas sobre o sexo, idade, escolaridade e questões relacionadas ao uso de plantas medicinais, abordando a origem da planta, a fonte do conhecimento popular, espécies utilizadas, indicação de uso, partes vegetais utilizadas, forma de preparo e frequência de uso. Quando disponíveis e com o consentimento dos entrevistados, as amostras com flores ou frutos foram coletadas e levadas para o herbário para serem herborizadas, montadas e procedida a identificação botânica. As exsicatas encontram-se depositadas no Herbário de Mafra, SC (HMSC), da Universidade do Contestado, campus de Mafra. Entretanto, nem todos os entrevistados puderam dispor de amostras férteis para a identificação. Por isso, essas plantas foram determinadas até o Gênero presuntivo, por comparação com fotos disponíveis na literatura. Após a coleta dos dados etnobotânicos, foram realizadas buscas na literatura sobre as espécies mencionadas pelos entrevistados a fim de verificar se há documentação de suas propriedades medicinais para posterior orientação à comunidade. 
Figura 1 - Município de Mafra (SC), área da pesquisa desenvolvida.

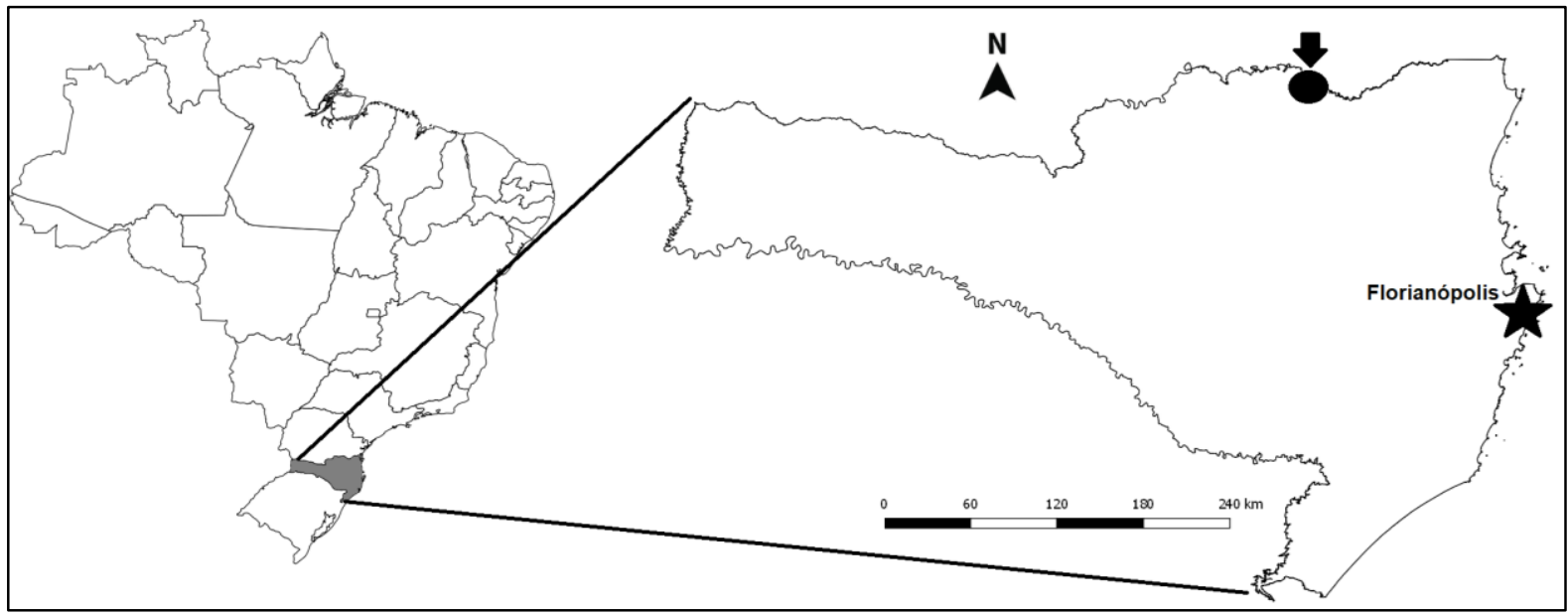

Fonte: Dados da pesquisa (2019).

A pesquisa foi realizada com anuência do Comitê de Ética em Pesquisa (CEP) da Universidade do Contestado, registrada com o Parecer número 3.304.931.

\section{RESULTADOS}

Dos 22 entrevistados, foi observado a prevalência de participantes do sexo feminino (86,36 \%) (Figura 2). Além disso, a maior parte dos participantes possuem mais de 60 anos de idade (36,36\%) e ensino fundamental incompleto (22,72\%) (Figura 2 ). Durante as entrevistas, alguns participantes mencionaram que o conhecimento acerca de plantas medicinais foi repassado pela figura materna. 
Figura 2 - (a) sexo; (b) idade; (c) escolaridade.

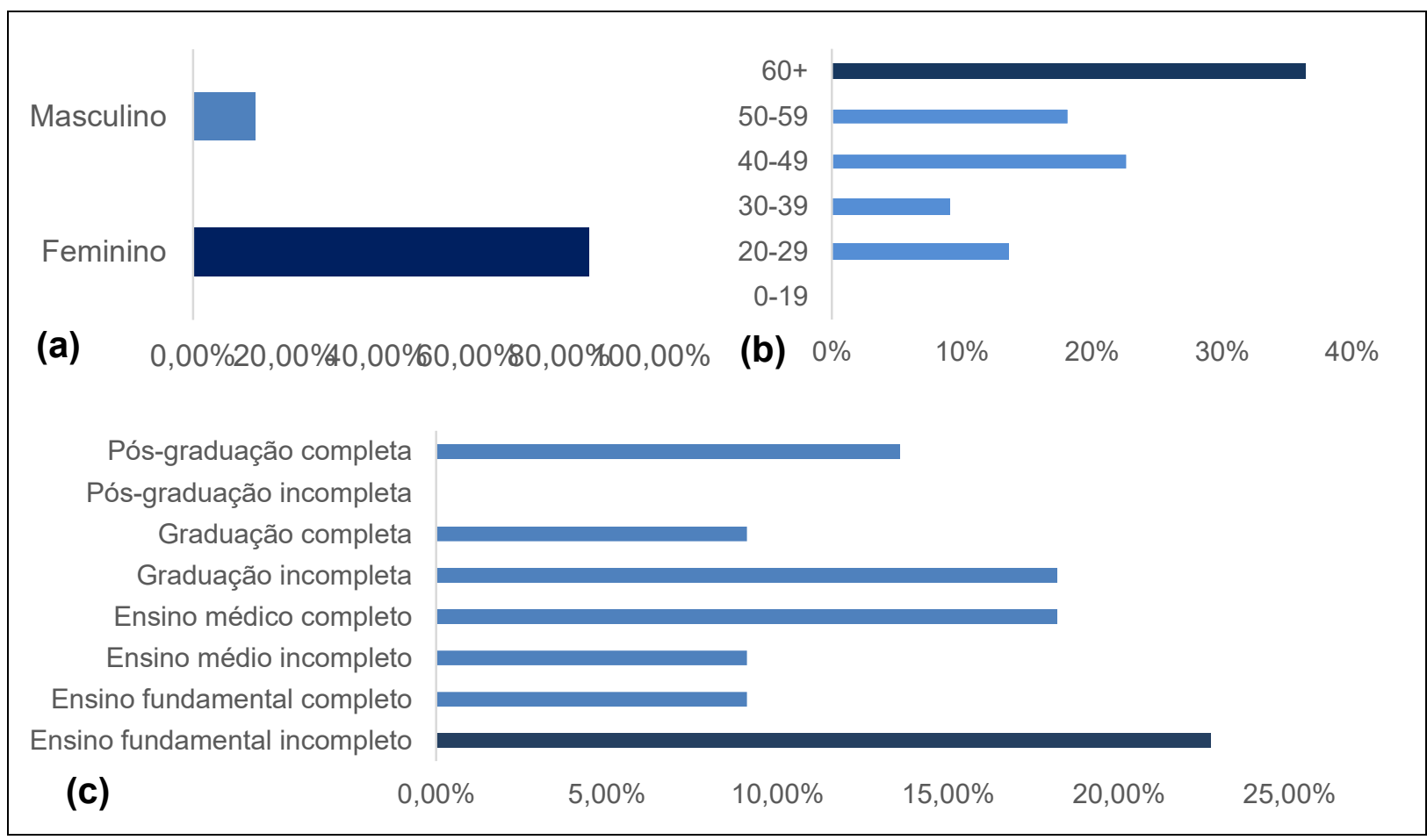

Fonte: Dados da pesquisa (2019).

A principal frequência de consumo dos usuários foi quando sente necessidade do uso $(60 \%)$, tendo como fonte do conhecimento popular sobre o uso das plantas familiares e amigos (37\%) (Figura 3). A origem predominante das espécies medicinais vem por meio do cultivo próprio (37\%) e o principal motivo do uso de plantas com finalidade terapêutica foi por ser natural (36\%) (Figura 3). Ainda com relação ao motivo de uso, $11 \%$ acredita que o uso de plantas medicinais não traz prejuízos à saúde dos usuários (Figura 3). Alguns informantes $(18,18 \%$ ) relataram o aparecimento de efeitos adversos com o uso de algumas espécies, conhecidas como salsa (Petroselinum sativum), guaco (Mikania sp.), capim-limão (Cymbopogon citratus) e moringa (Moringa sp.), que foram responsáveis, respectivamente, pelo efeito abortivo, tremor nas mãos, interrupção da menstruação e hipotensão. 
Figura 3 - (a) fonte do conhecimento popular sobre o uso de plantas medicinais; (b) frequência do consumo de plantas medicinais; (c) origem das espécies medicinais; (d) motivo pelo qual utiliza plantas com fins medicinais.

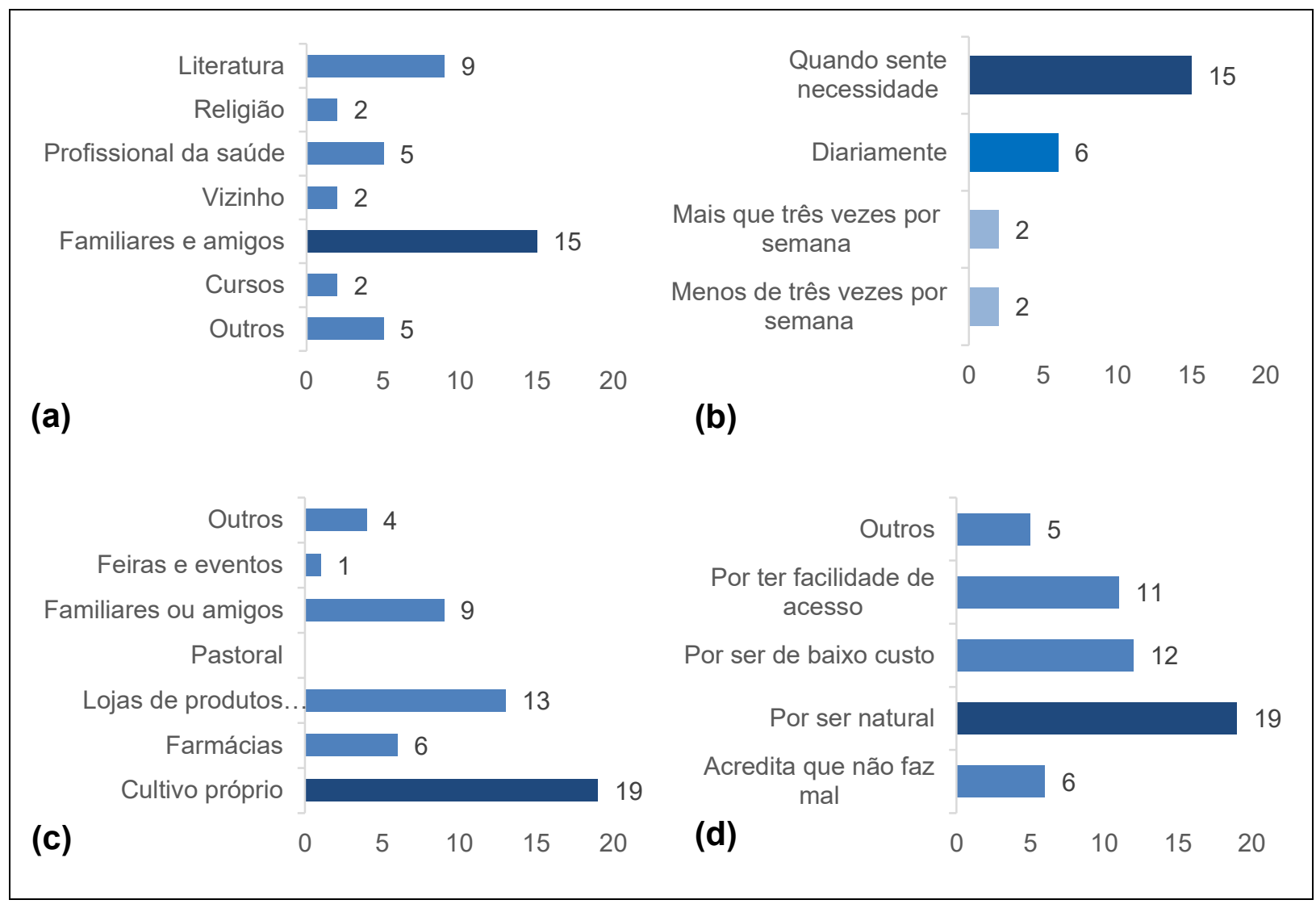

Fonte: Dados da pesquisa (2019).

Com relação aos dados etnobotânicos obtidos através das entrevistas com a população de Mafra, foram mencionadas 87 plantas no total, distribuídas em 49 famílias. As famílias predominantes foram Asteraceae (10 espécies citadas) e Lamiaceae (9 espécies citadas) (Tabela 1). As plantas mais citadas foram a babosa (Aloe spp.), a camomila (Chamomilla recutita), o capim-limão (Cymbopogon citratus), o gervão (Stachytarpheta spp.), a hortelã (Mentha spp.) e a penicilina (Alternanthera brasiliana) (Tabela 2).

Tabela 1 - Famílias botânicas predominantes.

\begin{tabular}{l|r}
\hline Família & Número de espécies \\
\hline Asteraceae & $10(11,5 \%)$ \\
Lamiaceae & $9(10,3 \%)$ \\
Myrtaceae & $5(5,7 \%)$ \\
Amaranthaceae & $4(4,6 \%)$ \\
Verbenaceae & $3(3,4 \%)$ \\
Rutaceae & $3(3,4 \%)$ \\
Rosaceae & $3(3,4 \%)$ \\
Malvaceae & $3(3,4 \%)$ \\
\hline
\end{tabular}

Fonte: Dados da pesquisa (2019). 
Outras plantas também foram mencionadas: alcachofra, açafrão, alfa-vaca anis, alho, ameixa, araçá, arruda, avenca, azedinha, bálsamo, bardana, batata-doce, burrito, cana-brava (caninha), calêdula, canela, canela-de-velho, caraguatá, carobinha-branca, caatinga-de-mulata, chá-verde, chuchu, cipó-milome (cipó-milhomens), coco, couve, dente-de-leão, endro, erva-do-brejo (chapéu-de-couro), ervamate, fáfia, figatil, folha de abacate, folha-gorda, gingko biloba, ginseng, goiaba, graviola, guanxumba, iacon (yacon), jabuticaba, kava-kava, lavanda, leiteira-africada (teborna ou janaúba), limão, losna, mamica-de-cadela, manjericão, malva, maracujá, melaleuca, mentruz, milho, moringa, mostarda, mulungu, pau-pelado, pêssego, picopico, plantago (tanchagem), salsa, uva, uxi-amarelo (unha-de-gato) e vique. 
Tabela 2 - Nome popular (NP) das plantas mais relatadas na pesquisa, nomes científicos (NC), família botânica, número de citações (NCt), parte da planta utilizada (PPU), indicação popular (IP) e indicação da literatura científica (ILC).

\begin{tabular}{|c|c|c|c|c|c|c|}
\hline NP & NC & Família & $\mathbf{N C t}$ & PPU & IP & ILC \\
\hline Alecrim & Rosmarinus officinalis L. & Lamiaceae & 3 & $\begin{array}{l}\text { partes } \\
\text { aéreas }\end{array}$ & $\begin{array}{l}\text { Estimulante } \\
\text { metabólico, gripe, } \\
\text { infecção na faringe, } \\
\text { bronquites, melhora da } \\
\text { circulação sanguínea } \\
\text { e batimentos } \\
\text { cardíacos }\end{array}$ & $\begin{array}{l}\text { hepatopatias, colecistites crônicas, } \\
\text { flatulência, edema associado a } \\
\text { hepatopatias (diurético), cólicas } \\
\text { menstruais e abdominais, tônico } \\
\text { geral, irregularidades menstruais, } \\
\text { artroses (aplicação local) (23) }\end{array}$ \\
\hline Amora & Rubus sp. & Rosaceae & 3 & folhas & $\begin{array}{l}\text { distúrbios } \\
\text { calmante, mormais, } \\
\text { pele e cabelos, } \\
\text { antidepressivo, } \\
\text { hipertensão e diabetes }\end{array}$ & $\begin{array}{lr}\begin{array}{l}\text { antioxidante, } \\
\text { antiestresse, } \\
\text { antimicrobiano, }\end{array} & \begin{array}{r}\text { antidiabético, } \\
\text { neuroprotetor, } \\
\text { anticâncer, } \\
\text { hepatoprotetor, } \\
\text { imunomoduladornico, } \\
(24)\end{array} \\
\text { a e anti-helmíntico, } \\
\text { dislipidemias }\end{array}$ \\
\hline Babosa & Aloe vera (L.) Burm. f. & Xanthorrhoeaceae & 4 & $\begin{array}{l}\text { folhas e } \\
\text { resina }\end{array}$ & $\begin{array}{lr}\text { queimadura, } & \\
\text { cicatrizante, } & \text { anti- } \\
\text { inflamatório } & \mathrm{e} \\
\text { anticâncer } & \end{array}$ & $\begin{array}{l}\text { cicatrizante e reconstituinte em } \\
\text { lesões térmicas (isquemia e } \\
\text { queimaduras), } \\
\text { (queimaduras, radioativas } \\
\text { telangiectasia), emoliente em } \\
\text { psoríase, eczemas, dermatites } \\
\text { seborreicas, furúnculos, hemorroida } \\
\text { e fissura anal (23) }\end{array}$ \\
\hline Boldo & Coleus forskohlii (Willd.) Briq. & Lamiaceae & 4 & folhas & $\begin{array}{l}\text { problemas do trato } \\
\text { gastrointestinal }\end{array}$ & $\begin{array}{l}\text { indigestão, hepatite, litíase biliar, } \\
\text { colecistite, náuseas e vômitos, } \\
\text { constipação intestinal (23) }\end{array}$ \\
\hline Camomila & Matricaria chamomilla L. & Asteraceae & 8 & flores & $\begin{array}{l}\text { anti-inflamatório, } \\
\text { ansiolítico, relaxante } \\
\text { muscular, } \\
\text { dismenorreia, } \\
\text { digestivo, prisão de } \\
\text { ventre }\end{array}$ & $\begin{array}{lr}\begin{array}{l}\text { sedativa, } \\
\text { antiespasmótica, } \\
\text { inflamação cutânea (23) }\end{array} & \text { dermatite, }\end{array}$ \\
\hline Capim-limão & $\begin{array}{l}\text { Cymbopogon citratus (DC.) } \\
\text { Stapf }\end{array}$ & Poaceae & 7 & folhas & $\begin{array}{l}\text { ansiolítico, } \\
\text { dismenorreia, }\end{array}$ & $\begin{array}{l}\text { antiespasmótica; } \\
\text { analgésica (23) }\end{array}$ \\
\hline
\end{tabular}




\begin{tabular}{|c|c|c|c|c|c|c|}
\hline & & & & & $\begin{array}{l}\text { relaxante muscular, } \\
\text { insônia, digestivo e } \\
\text { cefaleia }\end{array}$ & \\
\hline Cavalinha & Equisetum spp. & Equisetaceae & 4 & $\begin{array}{l}\text { partes } \\
\text { aéreas }\end{array}$ & $\begin{array}{l}\text { diurético, } \begin{array}{l}\text { anti- } \\
\text { inflamatório, favorece } \\
\text { a circulação }\end{array}\end{array}$ & $\begin{array}{l}\text { diurético em distúrbios do aparelho } \\
\text { urinário e próstata, edema pré- } \\
\text { menstrual associado ao estigma de } \\
\text { milho, remineralizante, como tônica } \\
\text { usada também no tratamento de } \\
\text { enurese noturna, antilítica (23) }\end{array}$ \\
\hline Erva-doce & $\begin{array}{l}\text { Pimpinella anisum } \mathrm{L} . \\
\text { Foeniculum vulgare Mill. }\end{array}$ & Apiaceae & 4 & $\begin{array}{l}\text { semente, } \\
\text { folhas e } \\
\text { galhos }\end{array}$ & $\begin{array}{l}\text { indigestão, } \\
\text { expectorante, dor de } \\
\text { ouvido, calmante, } \\
\text { cólica intestinal }\end{array}$ & $\begin{array}{l}\begin{array}{l}\text { antimicrobiano, } \\
\text { antiviral, antioxidante, } \\
\text { muscular, } \\
\text { hipoglicêmico, } \\
\text { analgésicóngico, } \\
\text { anticonvulsivante e neuroprotetor, } \\
\text { dismenorreia e sintomas da }\end{array} \\
\begin{array}{l}\text { menopausa; não há evidências do } \\
\text { efeito ansiolítico (25) }\end{array}\end{array}$ \\
\hline Espinheira-Santa & $\begin{array}{l}\text { Maytenus ilicifolia } \text { Mart. ex } \\
\text { Reissek }\end{array}$ & Celastraceae & 3 & folhas & $\begin{array}{l}\text { diurético, depurativo, } \\
\text { dislipidemias, } \\
\text { problemas no } \\
\text { estômago, cólica }\end{array}$ & 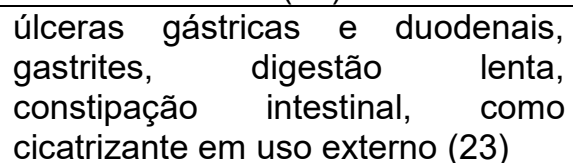 \\
\hline Eucalipto & Eucalyptus glubulus Labill. & Myrtaceae & 3 & $\begin{array}{l}\text { folhas e } \\
\text { caule }\end{array}$ & $\begin{array}{l}\text { descongestionante, } \\
\text { gripe, ansiolítico }\end{array}$ & $\begin{array}{l}\text { gripes, resfriado e febre, tosse } \\
\text { produtiva, sinusite, amigdalite, } \\
\text { bronquite, infecção urinária, cistite, } \\
\text { uretrite e leucorreias; uso externo: } \\
\text { cicatrizante de feridas; cataplasma } \\
\text { em inflamações de pele e contusões } \\
\text { e dores (23) }\end{array}$ \\
\hline Gengibre & Zingiber officinale Roscoe & Zingiberaceae & 3 & raiz & $\begin{array}{l}\text { anti-inflamatório, } \\
\text { antioxidante, acelera o } \\
\text { metabolismo, gripe, } \\
\text { dores musculares, } \\
\text { sinusite }\end{array}$ & $\begin{array}{l}\text { gripe, resfriados, tosse, bronquites, } \\
\text { irritação e inflamação da garganta, } \\
\text { rouquidão, digestão lenta, plenitude } \\
\text { pós-prandial, gastriter e } \\
\text { epigastralgias, flatulência, refluxo } \\
\text { gastroesofágico, intoxicação } \\
\text { alimentar (frutos do mar), doenças } \\
\text { reumáticas, dores e processos } \\
\text { inflamatórios articulares, como }\end{array}$ \\
\hline
\end{tabular}

Saúde Meio Ambient. v. 9, p. 27-42, 2020 


\begin{tabular}{|c|c|c|c|c|c|c|}
\hline & & & & & & $\begin{array}{l}\text { tônico e afrodisíaco, náuseas, } \\
\text { vômitos pós-cirurgicos, cinetose, } \\
\text { hiperêmese gravídica (23) }\end{array}$ \\
\hline Gervão & Stachytarpheta sp. & Verbenaceae & 5 & folhas & indigestão, diabetes & $\begin{array}{l}\text { Antibacteriano e antiespasmótico } \\
(26)\end{array}$ \\
\hline Guaco & Mikania glomerata Spreng. & Asteraceae & 4 & folhas & $\begin{array}{l}\text { tosse, dor de garganta, } \\
\text { bronquite, asma, gripe }\end{array}$ & $\begin{array}{l}\text { febres, gripes e resfriados, tosses } \\
\text { rebeldes, bronquite, asma, } \\
\text { faringites, laringites, rouquidão, } \\
\text { eczemas, gargarejos em infecções } \\
\text { de garganta, em loções no eczema } \\
\text { pruriginoso (uso externo) (23) }\end{array}$ \\
\hline Hibisco & Hibiscus sp. & Malvaceae & 3 & flores & diurético, termogênico & $\begin{array}{l}\text { antioxidante, anti-inflamatório, anti- } \\
\text { hipertensivo, problemas no fígado, } \\
\text { diabetes, síndrome metabólica, } \\
\text { dislipidemias, anti-obesidade, } \\
\text { anticoagulante, diurético, anti- } \\
\text { urolítico, antimicrobiano, anticâncer, } \\
\text { hepatoprotetor, antitumoral e } \\
\text { imunomodulador (27) }\end{array}$ \\
\hline Hortelã & $\begin{array}{l}\text { Mentha arvensis } \mathrm{L} . \\
\text { Mentha } x \text { piperita } \mathrm{L} . \\
\text { Mentha spicata } \mathrm{L} .\end{array}$ & Lamiaceae & 10 & folhas & $\begin{array}{l}\text { problemas } \\
\text { estomacais, } \\
\text { antiparasitário, } \\
\text { calmante }\end{array}$ & $\begin{array}{l}\text { resfriados e gripes, faringite, } \\
\text { amigdalite, rinite alérgica, asma } \\
\text { brônquica, bronquites e sinusites } \\
\text { (favorece a expectoração), } \\
\text { dispepsias em geral, cólicas } \\
\text { abdominais, flatulência, síndrome } \\
\text { do intestino irritável (23) }\end{array}$ \\
\hline Laranjeira & Citrus sp. & Rutaceae & 3 & $\begin{array}{l}\text { folhas e } \\
\text { fruto }\end{array}$ & $\begin{array}{l}\text { gripe, dor de garganta } \\
\text { e diarreia }\end{array}$ & $\begin{array}{l}\text { Folhas: gripes e resfriados, tosse } \\
\text { com expecoração amarelada e } \\
\text { abundante, resfriados de repetição. } \\
\text { Frutos: dispneia e palpitações. Fruto } \\
\text { e epicarpo: antiespasmótico, } \\
\text { camrinativo, halitose, gosto amargo } \\
\text { na boca, digestão lenta, } \\
\text { regurgitação de alimentos, dor, } \\
\text { distensão e plenitude abdominal, } \\
\text { borborigmos, constipação intestinal, } \\
\text { gastralgias, dispepsias e }\end{array}$ \\
\hline
\end{tabular}

Saúde Meio Ambient. v. 9, p. 27-42, 2020 


\begin{tabular}{|c|c|c|c|c|c|c|}
\hline & & & & & & $\begin{array}{l}\text { perturbações digestivas em geral. } \\
\text { Flores: ansiedades, histerias, } \\
\text { palpitações. Folhas: reumatismo e } \\
\text { taquicardia (23) }\end{array}$ \\
\hline Levante/Alevante & Mentha $x$ piperita $\mathrm{L}$. & Lamiaceae & 4 & folhas & desconforto estomacal & $\begin{array}{l}\text { dispepsia, flatulência, } \\
\text { intestinal, cólica } \\
\text { enterites, síndrome do intestino } \\
\text { irritável (23) }\end{array}$ \\
\hline Melissa & Melissa sp. & Lamiaceae & 3 & folhas & $\begin{array}{l}\text { calmante, gripe, } \\
\text { antimicrobiano, } \\
\text { calmante e distúrbios } \\
\text { do sono }\end{array}$ & $\begin{array}{l}\text { depressão, insônia, ansiedade e } \\
\text { antimicrobiano (28) e (29) }\end{array}$ \\
\hline Ora-pro-nóbis & Pereskia aculeata Miller. & Cactaceae & 3 & folhas & $\begin{array}{l}\text { complemento } \\
\text { alimentar (fonte de } \\
\text { proteínas), aumenta a } \\
\text { imunidade, varizes, } \\
\text { câncer de cólon, } \\
\text { hemorroidas, } \\
\text { furúnculos, } \\
\text { hiperlipidemias }\end{array}$ & 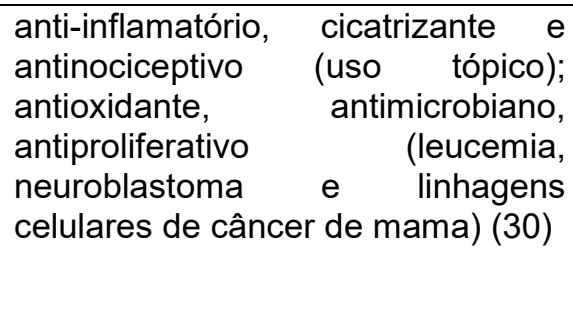 \\
\hline Pata-de-vaca & Bauhinia forficata Link & Fabaceae & 4 & folhas & $\begin{array}{lr}\text { diabetes, } & \text { cálculos } \\
\text { renais, } & \text { acnes, } \\
\text { diurética, dislipidemias }\end{array}$ & diabetes mellitus tipo 2 (23) \\
\hline Penicilina & Alternanthera sp. & Amaranthaceae & 6 & folhas & $\begin{array}{l}\text { anti-hipertensivo, anti- } \\
\text { inflamatório, diurético, } \\
\text { dores musculares }\end{array}$ & $\begin{array}{l}\text { antinociceptivo, antimicrobiano, } \\
\text { anti-herpes, bloqueia a proliferação } \\
\text { de linfócitos induzida por mitógeno } \\
\text { humano sem qualquer efeito tóxico } \\
\text { (31) }\end{array}$ \\
\hline Ponto-alívio & Achillea millefolium L. & Asteraceae & 3 & folhas & $\begin{array}{ll}\text { analgésico } & \mathrm{e} \\
\text { expectorante }\end{array}$ & $\begin{array}{l}\text { Inapetência, cólicas intestinais, } \\
\text { dispepsia, resfriados e gripes (23) }\end{array}$ \\
\hline Quebra-pedra & Phyllanthus spp. & Phyllanthaceae & 3 & folhas & $\begin{array}{l}\text { diurético, cálculos } \\
\text { renais e dislipidemias }\end{array}$ & $\begin{array}{l}\text { litíase renal e cólica nefrética, } \\
\text { hepatite } B(23)\end{array}$ \\
\hline
\end{tabular}

Fonte: dados da pesquisa (2019). 


\section{DISCUSSÃO}

Com relação ao sexo, a idade e a escolaridade dos participantes, resultados similares foram encontrados em outros estudos brasileiros(7),(10),(11). Referente a prevalência de conhecimento repassado pela figura materna, alguns levantamentos etnobotânicos realizados em alguns países do continente Asiático(12),(13)(14), apresentaram maior prevalência de informantes do sexo masculino. Esses dados mostram que, nesse continente, os homens possuem mais conhecimento sobre plantas devido ao fato de que existiam mais curandeiro do sexo masculino, diferentemente do Brasil, onde, antigamente, as mulheres eram cuidadoras da casa e da família.

Um estudo(6) relatou que a maior parte dos informantes $(75 \%)$ obteve seus conhecimentos tradicionais através de familiares, resultado semelhante ao do presente estudo. Esse conhecimento passado de geração para geração é essencial, pois significa que parte da cultura se manteve preservada. Entretanto, ainda é necessário que a população conheça os possíveis riscos do uso incorreto de plantas medicinais, como as interações planta-planta e entre planta-medicamento.

Além de apresentar maior frequência de conhecimentos obtidos hereditariamente, outro estudo ${ }^{(15)}$ relatou o uso seguro como o principal motivo para o uso de plantas medicinais pela população. A crença popular sobre "o que é natural, não faz mal" citado por alguns participantes não é verdadeira. Com os avanços de pesquisas na área, é possível verificar que em fitocomplexos presentes em uma planta in natura, mesmo que conhecida popularmente, existem metabólitos secundários que não apresentam benefícios ou que podem ser prejudiciais para o organismo. Vários estudos $^{(16),(21),(22)}$ sobre determinadas espécies demonstraram potencial tóxico ou danoso à saúde, por exemplo o uso do extrato de alecrim (Rosmarinus officinalis L.) que pode causar dermatite alérgica de contato, causado pelo constituinte diterpeno

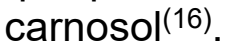

Os efeitos adversos causados por salsa (Petroselinum sativum), guaco (Mikania sp.), capim-limão (Cymbopogon citratus) e moringa (Moringa sp.) mencionados pelos participantes podem ser esclarecidos devido a presença de alguns constituintes químicos presentes nessas espécies, responsáveis por esses efeitos. Dessa forma, o efeito causado pelo uso de guaco (Mikania sp.), pode ser devido ao efeito hipoglicemiante dos compostos cumarina, escopoletina e acetato de lupeol ${ }^{(18)}$, enquanto que o efeito adverso do capim-limão (C. citratus) pode ter sido resultado da inibição da contração do útero induzida pela ocitocina ${ }^{(19)}$, ao passo que o efeito da moringa (Moringa sp.) pode ser resultado dos compostos nitreto, glicosídeos de óleo de mostarda e glicosídeos de tiocarbamato que são considerados responsáveis pelo efeito hipotensivo da planta ${ }^{(20)}$, e o uso das folhas frescas da salsa ( $P$. sativum) pode resultar em efeito abortivo(17).

As famílias prevalentes Asteraceae e Lamiaceae apresentadas nessa pesquisa foram as mesmas relatadas como predominantes em trabalhos feitos nos estados de Santa Catarina(7), do Rio Grande do $\operatorname{Sul}^{(21)}$ e do Rio de Janeiro(22), regiões também 
pertencentes ao bioma Mata Atlântica. Esses resultados similares podem ser resultado da similaridade da vegetação entre essas regiões. Com relação a indicação popular, a maioria das prováveis espécies possuem a mesma indicação encontrada na literatura científica, como apresentado na Tabela 2.

\section{CONCLUSÃO}

Com esses resultados foi possível resgatar uma parcela cultural referente ao conhecimento popular sobre plantas passado hereditariamente no município de Mafra (SC). Além disso, as entrevistas realizadas neste trabalho possibilitaram verificar que a maioria das plantas utilizadas por moradores apresentam embasamento científico em relação a indicação de uso. Desta forma, o presente estudo apresenta relevância na área de saúde pública e permitiu aproximar a Universidade com a população, onde conhecimentos científicos e populares foram trocados. No entanto, sugere-se mais estudos na área, como a realização de mais entrevistas, assim como a identificação botânica de espécies cultivadas pela população para o aprimoramento dos dados etnobotânicos.

\section{REFERÊNCIAS}

1. Dutra RC, Campos MM, Santos ARS, Calixto JB. Medicinal plants in Brazil: Pharmacological studies, drug discovery, challenges and perspectives. Pharmacol Res. 2016 Oct 1; 112:4-29.

2. Beltreschi L, de Lima RB, da Cruz DD. Traditional botanical knowledge of medicinal plants in a "quilombola" community in the Atlantic Forest of northeastern Brazil. Environ Dev Sustain. 2019 Jun 11; 21(3):1185-203.

3. Baldauf C, Kubo RR, Silva F, Irgang BE. Ferveu, queimou o ser da erva: conhecimentos de especialistas locais sobre plantas medicinais na região Sul do Brasil. Rev Bras Plantas Med. 2009; 11(3):282-91.

4. Simões CMO, Schenkel EP, Mello JCP, LA Mentz, Petrovick PR. Farmacognosia: Do Produto Natural ao Medicamento. 3 ed. Porto Alegre: Artmed; 2001.

5. Pinto ALA, Matos MJ, Moura Rufino MDS, Rufino MSM. O conhecimento etnográfico dos Tremembé da Barra do Mundaú, Ceará. Interações. 2018 Feb 16;19(1):15.

6. Ribeiro RV, Bieski IGC, Balogun SO, Martins DTO. Ethnobotanical study of medicinal plants used by Ribeirinhos in the North Araguaia microregion, Mato Grosso, Brazil. J Ethnopharmacol. 2017 Jun 9; 205:69-102. 
7. Tribess B, Pintarelli GM, Bini LA, Camargo A, Funez LA, De Gasper AL, et al. Ethnobotanical study of plants used for therapeutic purposes in the Atlantic Forest region, Southern Brazil. J Ethnopharmacol. 2015 Apr 22; 164:136-46.

8. Sevegnani L, Schroeder E. Biodiversidade catarinense: características, potencialidades, ameaças. Blumenau: Edifurb; 2013.

9. Intituto Brasileiro de Geografia e Estatística. Cidades e Estados: Mafra. 2018. Available from: https://www.ibge.gov.br/cidades-e-estados/sc/mafra.html

10. Meyer L, Quadros KE, Zeni ALB. Revista Brasileira de biociências. Brazilian journal of biosciences. 2012; 10:258-66.

11. Maia EA, Francisco J, Pires T, Manfredi-Coimbra S. O uso de espécies vegetais para fins medicinais por duas comunidades da Serra Catarinense, Santa Catarina, Brasil. 2011; 11:54-74.

12. Panmei R, Gajurel PR, Singh B. Ethnobotany of medicinal plants used by the Zeliangrong ethnic group of Manipur, northeast India. J Ethnopharmacol. 2019 May 10; 235:164-82.

13. Yaseen G, Ahmad M, Sultana S, Suleiman AA, Hussain J, Zafar M, et al. Ethnobotany of Medicinal Plants in the Thar Desert (Sindh) of Pakistan. J Ethnopharmacol. 2015 Apr 2; 163:43-59.

14. Kayani S, Ahmad M, Sultana S, Khan SZ, Zafar M, Yaseen G, et al. Ethnobotany of medicinal plants among the communities of Alpine and Sub-alpine regions of Pakistan. J Ethnopharmacol. 2015 Apr 22; 164:186-202.

15. Bieski IGC, Leonti M, Arnason JT, Ferrier J, Rapinski M, Violante IMP, et al. Ethnobotanical study of medicinal plants by population of Valley of Juruena Region, Legal Amazon, Mato Grosso, Brazil. J Ethnopharmacol. 2015 Sep 15; 173:383-423.

16. Miroddi M, Calapai G, Isola S, Minciullo PL, Gangemi S. Rosmarinus officinalis L. as cause of contact dermatitis. Allergol Immunopathol (Madr). 2014 Nov 1; 42(6):616-9.

17. Cornara L, La Rocca A, Terrizzano L, Dente F, Mariotti MG. Ethnobotanical and phytomedical knowledge in the North-Western Ligurian Alps. J Ethnopharmacol. 2014 Aug 8; 155(1):463-84.

18. Marles RJ, Farnsworth NR. Antidiabetic plants and their active constituents. Vol. 2, Phytomedicine. 1995. p. 137-89.

19. Wei $\mathrm{Y}$, Ma T, Wang $\mathrm{H}$, Xing J, Wang $\mathrm{Y}, \mathrm{Gu} Z$, et al. Extracts of compound Muniziqi granule suppressed uterus contraction and ameliorated oxytocin-induced primary dysmenorrhea. J Ethnopharmacol. 2018 Sep 15; 223:33-40. 
20. Abrogoua DP, Dano DS, Manda P, Adepo AJB, Kablan BJ, Goze NB, et al. Effect on blood pressure of a dietary supplement containing traditional medicinal plants of Côte d'Ivoire. J Ethnopharmacol. 2012 Jun 14; 141(3):840-7.

21. Stolz ED, Müller LG, Trojan-Rodrigues M, Baumhardt E, Ritter MR, Rates SMK. Survey of plants popularly used for pain relief in Rio Grande do Sul, southern Brazil. Brazilian J Pharmacogn. 2014; 24(2):185-96.

22. Leitão F, Leitão SG, da Fonseca-Kruel VS, Silva IM, Martins K. Medicinal plants traded in the open-air markets in the State of Rio de Janeiro, Brazil: An overview on their botanical diversity and toxicological potential. Brazilian J Pharmacogn. $2014 ; 24(2): 225-47$.

23. Saad GA, Léda PHO, Sá IM, Seixlack ACC. Fitoterapia contemporânea: Tradição e ciência na prática clínica. 2. ed. Rio de Janeiro: Guanabara Koogan; 2016.

24. Hussain F, Rana Z, Shafique H, Malik A, Hussain Z. Phytopharmacological potential of different species of Morus alba and their bioactive phytochemicals: $A$ review. Asian Pac J Trop Biomed. 2017 Oct 1; 7(10):950-6.

25. Pontes VCB, Rodrigues DP, Caetano A, Gamberini MT. Preclinical investigation of the cardiovascular actions induced by aqueous extract of Pimpinella anisum L. seeds in rats. J Ethnopharmacol. 2019 Jun 12; 237:74-80.

26. Okoye T, Akah P, Okoli C, Ezike A, Mbaoji F. Antimicrobial and antispasmodic activity of leaf extract and fractions of Stachytarpheta cayennensis. Asian Pac J Trop Med. 2010 Mar; 3(3):189-92.

27. Riaz G, Chopra R. A review on phytochemistry and therapeutic uses of Hibiscus sabdariffa L. Biomedicine and Pharmacotherapy. 2018 Jun; 102:575-86.

28. Abdel-Naime WA, Fahim JR, Fouad MA, Kamel MS. Antibacterial, antifungal, and GC-MS studies of Melissa officinalis. South African J Bot. 2019 Aug 1; 124:22834.

29. Soltanpour A, Alijaniha F, Naseri M, Kazemnejad A, Heidari MR. Effects of Melissa officinalis on anxiety and sleep quality in patients undergoing coronary artery bypass surgery: A double-blind randomized placebo controlled trial. Eur J Integr Med. 2019 Jun 1; 28:27-32.

30. Garcia JAA, Corrêa RCG, Barros L, Pereira C, Abreu RMV, Alves MJ, et al. Phytochemical profile and biological activities of "Ora-pro-nobis" leaves (Pereskia aculeata Miller), an underexploited superfood from the Brazilian Atlantic Forest. Food Chem. 2019 Oct 1; 294:302-8.

31. Enechi OC, Odo CE, Wuave CP. Evaluation of the in vitro anti-oxidant activity of Alternanthera brasiliana leaves. J Pharm Res. 2013 Sep; 6:919-24. 


\section{AGRADECIMENTOS}

Agradecemos a todos os participantes que auxiliaram o levantamento dos dados etnobotânicos, a Universidade do Contestado pelo apoio financeiro (Fundo de Apoio a Pesquisa - FAP), além de todos os funcionários, profissionais e amigos que contribuíram na realização desse trabalho.

Artigo recebido em: 23/10/2019

Artigo aprovado em: 27/03/2020

Artigo publicado em: 30/03/2020 